\title{
Desafios na utilização da caderneta de saúde para o diagnóstico de crianças com Transtorno do Espectro Autista: uma revisão integrativa
}

Desafios na utilização da caderneta de saúde para o diagnóstico de crianças com Transtorno do Espectro Autista: uma revisão integrativa

Desafíos en el uso del folleto de salud para el diagnóstico de niños con Trastorno del Espectro Autista: una revisión integradora

Ana Tereza Santos Dias de Almeida ORCID: https://orcid.org/0000-0001-9927-1836 Centro Universitário de Ciências e Tecnologia do Maranhão, Brasil E-mail: aterezadias@gmail.com

Monyka Brito Lima dos Santos ORCID: https://orcid.org/0000-0002-6866-9435 Universidade Federal do Ceará, Brasil E-mail: monyka.brito@hotmail.com Karine Costa Melo

ORCID: https://orcid.org/0000-0001-8253-859X Universidade Federal do Maranhão, Brasil E-mail: karinemelo09@gmail.com

Wenderson Costa da Silva

ORCID: https://orcid.org/0000-0001-6031-9775

Universidade Estadual do Maranhão, Brasil

E-mail: wendersoncosta09@hotmail.com

Raylane Maria da Silva Rocha

ORCID: https://orcid.org/0000-0002-1956-8646

Universidade Estadual do Maranhão, Brasil

E-mail: raylanebiologa@gmail.com

Surama Almeida Oliveira

ORCID: https://orcid.org/0000-0002-9746-5765

Centro Universitário de Ciências e Tecnologia do Maranhão, Brasil

E-mail: su.a.oliveira@hotmail.com

Samara Ribeiro Farias

ORCID: https://orcid.org/0000-0001-5307-380X

Centro Universitário de Ciências e Tecnologia do Maranhão, Brasil

E-mail: oliveirasam03@gmail.com

Karen Lais Azevedo Oliveira Sousa

ORCID: https://orcid.org/0000-0002-0308-417X Centro Universitário de Ciências e Tecnologia do Maranhão, Brasil E-mail: karen.lais05@gmail.com

Eudilene da Silva Mesquita

ORCID: https://orcid.org/0000-0001-9894-0903 Centro Universitário de Ciências e Tecnologia do Maranhão, Brasil E-mail: eu.mesquita@gmail.com

Rayssa Stefani Cesar Lima ORCID: https://orcid.org/0000-0001-8859-7961

Universidade Estadual do Maranhão, Brasil E-mail: rayssastefany13@hotmail.com

Mayra Raisa Sena Sousa

ORCID: https://orcid.org/0000-0001-5519-1754 Centro Universitário de Ciências e Tecnologia do Maranhão, Brasil E-mail: Maraisasenna953@gmail.com

Layla Valéria Araújo Borges

ORCID: https://orcid.org/0000-0002-4715-407X

Universidade Estadual do Maranhão, Brasil E-mail: laylavaleria25@gmail.com

Natália Monteiro Pessoa

ORCID: https://orcid.org/0000-0001-6502-5661 Instituto de Pesquisas Ensino e Gestão em Saúde, Brasil

E-mail: natalia_tulip@hotmail.com 
Isadora Ravenna dos Santos Reis ORCID: https://orcid.org/0000-0002-3212-3166 Universidade Estadual do Maranhão, Brasil E-mail: isadoraravenna20@gmail.com

Alanna Nunes Soares

ORCID: https://orcid.org/0000-0002-0904-4515 Universidade Federal do Maranhão, Brasil E-mail: alanna_ns@hotmail.com

\begin{abstract}
Resumo
A Caderneta de Saúde da Criança (CSC) foi fundada como um meio gratuito de visualização e vigilância do crescimento e desenvolvimento infantil, onde o artigo traz como objetivo destacar os principais desafios enfrentados pelos profissionais de enfermagem na utilização da Caderneta de Saúde para o diagnóstico de crianças com TEA. Trata-se de uma pesquisa bibliográfica do tipo revisão integrativa de literatura, consistindo em uma pesquisa do tipo qualitativa, integrativa, exploratória e descritiva. Quanto aos resultados, os profissionais têm uma grande dificuldade em manejar a caderneta de saúde da criança, encontrando-se despreparados para prestar uma vigilância à saúde da criança com eficácia. Há inúmeros benefícios no uso de intervenção precoce em crianças com transtorno do espectro autista, entre eles destacam-se: Melhor desenvolvimento cognitivo e maior aprendizagem, redução do estresse para a criança e familiares e, maior interação social. Neste aspecto, ressalta-se a necessidade dos profissionais de enfermagem identificar ou colaborarem para identificação dessas crianças. Portanto, há uma necessidade de instruir profissionais e a coletividade sobre os benefícios da utilização da CSC além da realização de mais pesquisas nesta linha de investigação para comprovar a importância de uma atenção especial a saúde da criança.
\end{abstract}

Palavras-chave: Transtorno do Espectro Autista; Diagnóstico; Cuidados de saúde.

\begin{abstract}
The Child Health Handbook (CHH) was founded as a free means of visualization and surveillance of child growth and development, where the article aims to highlight the main challenges faced by nursing professionals in using the Health Handbook for the diagnosis of children with ASD. This is a bibliographic research of the integrative literature review type, consisting of a qualitative, integrative, exploratory and descriptive research. As for the results, professionals have great difficulty in handling the child's health booklet, finding themselves unprepared to effectively monitor the child's health. There are numerous benefits of using early intervention in children with autism spectrum disorder, including: Better cognitive development and greater learning, reduced stress for the child and family, and greater social interaction. In this aspect, the need for nursing professionals to identify or collaborate to identify these children is highlighted. Therefore, there is a need to instruct professionals and the community about the benefits of using $\mathrm{CHH}$, in addition to carrying out more research in this line of investigation to prove the importance of special attention to children's health.
\end{abstract}

Keywords: Autism Spectrum Disorder; Diagnosis; Health care.

\title{
Resumen
}

El Manual de Salud Infantil (MSI) fue fundado como un medio gratuito de visualización y vigilancia del crecimiento y desarrollo infantil, donde el artículo tiene como objetivo resaltar los principales desafíos que enfrentan los profesionales de enfermería en el uso del Health Handbook para el diagnóstico de niños con TEA. Se trata de una investigación bibliográfica del tipo revisión integradora de la literatura, consistente en una investigación cualitativa, integradora, exploratoria y descriptiva. En cuanto a los resultados, los profesionales tienen grandes dificultades para manejar el cuadernillo de salud del niño, encontrándose no preparados para monitorear eficazmente la salud del niño. Existen numerosos beneficios de utilizar la intervención temprana en niños con trastorno del espectro autista, que incluyen: Mejor desarrollo cognitivo y mayor aprendizaje, menor estrés para el niño y la familia y una mayor interacción social. En este aspecto, se destaca la necesidad de que los profesionales de enfermería identifiquen o colaboren para identificar a estos niños. Por tanto, es necesario instruir a los profesionales y a la comunidad sobre los beneficios del uso de MSI, además de realizar más investigaciones en esta línea de investigación para demostrar la importancia de una atención especial a la salud infantil.

Palabras clave: Trastorno del Espectro Autista; Diagnóstico; Cuidados de la salud. 


\section{Introdução}

A Caderneta de Saúde da Criança foi fundada como um meio gratuito de visualização e vigilância do crescimento e desenvolvimento infantil, comportando registros dos eventos mais importantes à saúde da criança do pré-natal ao prosseguimento de puericultura e constitui significativo meio de comunicação entre família e profissionais da saúde. Além disso, pertence à família, possibilitando que ela transite pelos diversos serviços de atenção à saúde e contribui para a coordenação do cuidado da criança (Rosolem et al., 2019).

O Transtorno do Espectro Autista (TEA) compõe-se por alterações no padrão de desenvolvimento neurológico. É perceptível que a partir do terceiro ano de vida a capacidade de socialização e de comunicabilidade é afetada, predispondo o sujeito a comportamentos estereotipados. Envolve aqueles que apresentem desde deficiência intelectual grave até indivíduos com quociente de inteligência padrão. A Organização Mundial da Saúde (OMS) caracteriza o TEA como uma alteração do desenvolvimento, irremediável e severamente incapacitante, com incidência de cinco casos a cada 100.000 nascidos vivos (Melo et al., 2019).

Acredita-se que o desenvolvimento neuropsicomotor começa já na vida intrauterina; a atividade reflexa se expressa pela motricidade, mas o processamento do aprendizado já é intensivo, pois compreendemos que todos os recém-nascidos precisam ser acompanhados até a idade escolar, para que se possa agir precocemente em qualquer imperfeição da aprendizagem, considerando a partir das funções primárias como a motricidade até as funções mais complexas como o desenvolvimento da linguagem (Fernandes, Vieira, Silva, Avelino, \& Santos, 2018).

Toda criança com retardo nas áreas sociais e da linguagem, devem ser encaminhadas à avaliação audiológica, se há casos de irmãos mais novos de crianças diagnosticadas com TEA, estes devem ser monitorados e todos os profissionais da atenção primária (pediatra, enfermeiro neurologista, psiquiatra, psicólogo, audiologista, fonoaudiólogo, terapeuta ocupacional e fisioterapeuta) devem estar adaptados com os sinais do TEA e investigar através da anamnese, vestígios que confirmem o diagnóstico.

Para tal a problemática da pesquisa foi "Quais os desafios na utilização correta da caderneta de saúde para o diagnóstico de crianças com transtorno do espectro autista?”. Onde o objetivo do estudo foi destacar os principais desafios enfrentados pelos profissionais de enfermagem na utilização da Caderneta de Saúde para o diagnóstico de crianças com TEA.

\section{Metodologia}

O estudo trata-se de uma pesquisa bibliográfica do tipo revisão integrativa de literatura, consistindo em uma pesquisa do tipo qualitativa, integrativa, exploratória e descritiva. De acordo com Paiva, Parente, Brandão e Queiroz (2016), esse modelo de revisão é o mais amplo campo de abordagem metodológica referente a revisões que faz o uso de estudos experimentais e não experimentais, para uma compreensão total do elemento posto em análise, além de permitir a realização de síntese dos resultados obtidos em pesquisas sobre um tema ou questão, de maneira sistemática, ordenada e abrangente. É denominada integrativa porque fornece informações mais amplas sobre um assunto/problema, constituindo, assim, um leque de conhecimento.

O tema "DESAFIOS NA UTILIZAÇÃO DA CADERNETA DE SAÚDE PARA O DIAGNÓSTICO DE CRIANÇAS COM TRANSTORNO DO ESPECTRO AUTISTA”, determinou a construção da estratégia PICo, que representa um acrônimo para Paciente $(\mathrm{P})$, Interesse $(\mathrm{I})$, Contexto $(\mathrm{Co})$ na qual foi utilizada para a geração da questão norteadora desta revisão integrativa da literatura: "Quais os desafios na utilização correta da caderneta de saúde para o diagnóstico de crianças com transtorno do espectro autista?". 
Para iniciar a busca e localização dos estudos relevantes, que respondessem à pergunta de pesquisa, utilizou-se de descritores indexados e não indexados (palavras-chave), nos idiomas português, inglês. Os descritores foram obtidos a partir do Medical Subject Headings (MESH), dos Descritores em Ciências da Saúde (DeCS), como mostra o Quadro 1.

Quadro 1. Elementos da estratégia PICO, descritores e palavras-chave utilizados. Caxias, MA, Brasil, 2020.

\begin{tabular}{|c|c|c|c|c|}
\hline \multicolumn{2}{|r|}{ Elementos } & Decs & Mesh & Palavras-chave \\
\hline $\mathbf{P}$ & $\begin{array}{l}\text { Transtorno do } \\
\text { Espectrodo Autismo }\end{array}$ & $\begin{array}{l}\text { “Autism Spectrum Disoder” } \\
\text { “Transtorno do Espectro } \\
\text { Autista” }\end{array}$ & $\begin{array}{c}\text { "Autism Spectrum } \\
\text { Disoder" }\end{array}$ & $\begin{array}{c}\text { "Autism Spectrum Disoder" } \\
\text { "Transtorno do Espectro do } \\
\text { Autimo" }\end{array}$ \\
\hline $\mathbf{I}$ & Rastreamento & $\begin{array}{l}\text { “Diagnosis” } \\
\text { “Diagnóstico" }\end{array}$ & "Diagnosis" & "Diagnosis" “Diagnóstico” \\
\hline Co & Intervenção & $\begin{array}{c}\text { "Outcome Assessment (Health } \\
\text { Care)” } \\
\text { “Avaliação de Resultados } \\
\text { (Cuidados de Saúde)” }\end{array}$ & $\begin{array}{c}\text { "Outcome Assessment } \\
\text { (Health Care)" }\end{array}$ & $\begin{array}{c}\text { "Outcome Assessment (Health } \\
\text { Care)” } \\
\text { “Avaliação de Resultados } \\
\text { (Cuidados de Saúde)” }\end{array}$ \\
\hline
\end{tabular}

Fonte: Descritores, Títulos e Palavras-chaves (2020).

Consultou-se por meio de descritores e palavras-chave as bases de dados PubMed da National Library of Medicine e BVS (Biblioteca Virtual da Saúde), coordenada pela BIREME e SicELO (Quadro 2).

Quadro 2. Estratégias de busca utilizadas nas bases de dados BIREME, PUBMED e SCIELO. Caxias, MA, Brasil, 2020.

\begin{tabular}{|c|c|c|c|c|}
\hline $\begin{array}{c}\text { Base de } \\
\text { dados }\end{array}$ & Estratérgia de Busca & Resultados & Filtrados & Selecionados \\
\hline $\begin{array}{c}\text { BIREME } \\
\text { (descritores } \\
\text { Decs) }\end{array}$ & $\begin{array}{l}\text { (tw:(Autismo)) OR (tw:(Rastreamento de crianças com Autismo)) } \\
\text { OR (tw:(Caderneta de Saúde da Criança)) }\end{array}$ & 34.152 & 344 & 1 \\
\hline $\begin{array}{c}\text { PUBMED } \\
\text { (descriptors } \\
\text { MeSH) }\end{array}$ & $\begin{array}{l}\text { (autismo[All Fields] OR (rastreamento[All Fields] AND ("drug } \\
\text { effects"[Subheading] OR ("drug"[All Fields] AND "effects"[All } \\
\text { Fields]) OR "drug effects"[All Fields] OR "de"[All Fields]) AND } \\
\text { criancas[All Fields] AND com[All Fields] AND autismo[All } \\
\text { Fields])) OR (caderneta[All } \quad \text { Fields] AND ("drug } \\
\text { effects"[Subheading] OR ("drug"[All Fields] AND "effects"[All } \\
\text { Fields]) OR "drug effects"[All Fields] OR "de"[All Fields]) AND } \\
\text { "saude"[All Fields] AND da[All Fields] AND crianca[All Fields]) } \\
\text { AND ("2015/04/28"[PDat]: "2020/04/25"[PDat]) }\end{array}$ & 107 & 70 & 2 \\
\hline SciELO & $\begin{array}{l}\text { (* autismo) OR (rastreamento em crianças com autismo) OR } \\
\text { (caderneta de saúde da criança) AND in:("scl") AND la:("pt") AND } \\
\text { year_cluster:("2015" OR "2018" OR "2014" OR "2017" OR "2016" } \\
\text { OR "2019") AND type:("research- } \\
\text { article") }\end{array}$ & 696 & 151 & 7 \\
\hline
\end{tabular}

Fonte: Bases de dados (2020).

Os critérios de inclusão definidos foram: estudos primários, disponíveis em sua totalidade, publicados entre os anos de 2014 até 2019, nos idiomas Português e Inglês. Foram excluídos da busca inicial capítulos de livros, resumos, textos incompletos, teses de doutorado, dissertações de mestrados, monografias e relatos técnicos.

A pesquisa foi realizada em três etapas, onde a primeira consistiu em uma análise das informações colhidas dos artigos científicos a qual se tornou necessária a criação de categorias analíticas para que fosse possível a ordenação e 
sumarização de cada estudo, sendo feita de forma descritiva, destacando os dados mais importantes a serem utilizados na pesquisa. O estudo levou em consideração os aspectos éticos da pesquisa quanto às citações dos estudos, respeitando a autoria das ideias, os conceitos e as definições presentes nos artigos incluídos na revisão.

Encontraram-se trinta e quatro mil e cento e cinquenta e dois (34.152) estudos como busca geral na BVS, sendo que limitando a busca para artigos com texto completo realizado com humanos nos últimos cinco anos, obteve-se trezentos e quarenta e quatro (344) estudos, destes foram analisados títulos e resumos onde apenas um (1) estudos foram condizentes com a questão desta pesquisa.

Na base PUBMED, como busca total foram encontrados cento e sete (107) estudos, aplicando na pesquisa o filtro que limita por texto completo dos últimos cinco anos com humanos, obteve-se setenta (70) estudos, destes foram analisados títulos e resumos e teve como resultado final de dois (2) estudos.

No ScIELO, encontrou-se como busca total seiscentos e noventa e seis (696), aplicando o filtro que limita por texto completo dos últimos cinco anos, realizados com humanos, obteve-se cento e cinquenta e um (151), e foram selecionados sete (7) sendo analisados por títulos, resumos, condizentes com a questão da pesquisa.

$\mathrm{Na}$ segunda fase os estudos foram analisados quanto ao potencial de participação no estudo, avaliando o atendimento à questão de pesquisa, bem como o tipo de investigação, objetivos, amostra, método, resultados e conclusão, resultando em dez (10) artigos.

A segunda etapa foi à realização da categorização dos estudos selecionados, utilizou-se um instrumento denominado de matriz de síntese ou matriz de análise, que permite analisar separadamente cada artigo, extrair e organizar os dados tanto num nível metodológico quanto em relação aos resultados das pesquisas. Tal instrumento possibilita a síntese dos artigos, salvaguardando suas diferenças, criando categorias analíticas que facilitem a ordenação e sumarização de cada estudo.

O instrumento contemplou itens como o nome do artigo, ano de publicação, autores, objetivo, perfil amostral e principais resultados. Neste estudo utilizou-se o instrumento validado por Urbanetto et al. (2017), que contempla variáveis de análise para a Prática Baseada em Evidências Científicas.

$\mathrm{Na}$ última etapa, foram analisadas as informações coletadas nos artigos científicos e criadas categorias analíticas que facilitou a ordenação e a sumarização de cada estudo. Essa categorização foi realizada de forma descritiva, indicando os dados mais relevantes para o estudo.

A pesquisa levou em consideração os aspectos éticos quanto às citações dos estudos, respeitado a autoria das ideias, os conceitos e as definições presentes nos artigos incluídos na revisão. Optou-se pela análise em forma estatística e de forma de texto, utilizando cálculos matemáticos e inferências, que estão apresentados em quadros e tabelas para facilitar a visualização e compreensão.

\section{Resultados e Discussão}

Os dez estudos que foram inclusos nesta revisão estavam nas diferentes bases de dados e na língua portuguesa e inglesa. As publicações foram concentradas nos anos de 2014 a 2019 com abordagem quantitativa, o nível de evidência predominante foi composto por estudos transversais, revisões integrativas e sistemáticas da literatura. O Brasil foi o país com mais estudos incluídos que atendiam aos objetivos propostos, conforme demonstrado na Tabela 1. 
Tabela 1. Análise descritiva das produções científicas acerca dos desafios na utilização da caderneta de saúde para o diagnóstico de crianças com transtorno do espectro autista. Caxias - MA, 2020. (n=10).

\begin{tabular}{|c|c|c|}
\hline VARIÁVEIS & $\mathbf{N}$ & $\%$ \\
\hline \multicolumn{3}{|l|}{ Abordagem do estudo } \\
\hline Qualitativo & 01 & 10 \\
\hline Quantitativo & 09 & 90 \\
\hline \multicolumn{3}{|l|}{ Delineamento da pesquisa } \\
\hline Revisão Sistemática & 02 & 20 \\
\hline Revisão Integrativa & 02 & 20 \\
\hline Estudo Transversal & 05 & 50 \\
\hline Pesquisa Descritiva & 01 & 10 \\
\hline \multicolumn{3}{|l|}{ Idioma } \\
\hline Inglês & 01 & 10 \\
\hline Português & 09 & 90 \\
\hline \multicolumn{3}{|l|}{ Procedência } \\
\hline Brasil & 09 & 90 \\
\hline EUA & 01 & 10 \\
\hline \multicolumn{3}{|l|}{ Distribuição Temporal } \\
\hline 2014 & 02 & 20 \\
\hline 2015 & 03 & 30 \\
\hline 2016 & 03 & 30 \\
\hline 2017 & 01 & 10 \\
\hline 2018 & 01 & 10 \\
\hline
\end{tabular}

Fonte: Elaboração própria (2020).

A tabela traz informações importantes sobre as produções cientificas acerca do tema abordado, possibilitando aos leitores observarem quais os anos que concentraram mais publicações (2014 a 2018). E a abordagem e o nível de evidência das publicações, além do tipo de estudo, na qual pode se afirmar que o Brasil foi o país com mais estudos relevantes ao tema proposto, conforme demonstrado na tabela. Por fim, o Quadro 3 traz outras informações importantes sobre os artigos escolhidos, como exemplo, o perfil amostral. 
Quadro 3. publicações incluídas segundo autor; tipo de estudo, objetivo principal, perfil amostral e intervenções/interesse. Caxias, MA, Brasil, 2020. (N=10).

\begin{tabular}{|c|c|c|c|c|}
\hline $\begin{array}{c}\text { Bases } \\
\text { de dados }\end{array}$ & $\begin{array}{c}\text { Autores } \\
\text { /ano }\end{array}$ & Objetivo & Perfil amostral & Principais resultados \\
\hline $\begin{array}{c}1 \\
\text { SCIELO }\end{array}$ & $\begin{array}{l}\text { Amorim } \\
\text { etal. } \\
(2018)\end{array}$ & $\begin{array}{l}\text { Descrever o preenchimento da } \\
\text { Caderneta de Saúde da Criança } \\
\text { (CSC) nos serviços de saúde. }\end{array}$ & $\begin{array}{l}\text { Estudo descritivo, desenvolvido em Belo Horizonte, } \\
\text { Minas Gerais, Brasil; realizou-se entrevista com os } \\
\text { pais/responsáveis de crianças de } 3 \text { a } 5 \text { anos de idade e } \\
\text { observaram-se } 21 \text { itens essenciais ao acompanhamento } \\
\text { infantil na CSC, no Dia de Multivacinação de } 2014 \text {; } \\
\text { considerou-se separadamente os campos a serem } \\
\text { preenchidos nas maternidades e na Atenção Primária à } \\
\text { Saúde (APS) e outros serviço }\end{array}$ & $\begin{array}{l}\text { O enfrentamento da precariedade nos registros da CSC passa } \\
\text { pela formação de profissionais de saúde que saibam reconhecer } \\
\text { a importância do instrumento para a vigilância da saúde } \\
\text { infantil, bem como do empoderamento dos pais ou } \\
\text { responsáveis, que devem portar e exigir o uso da CSC nos } \\
\text { diferentes serviços de saúde da criança. }\end{array}$ \\
\hline $\begin{array}{c}2 \\
\text { PUBMED }\end{array}$ & $\begin{array}{l}\text { Caminha } \\
\text { et al. } \\
(2017)\end{array}$ & $\begin{array}{l}\text { Descrever o caso do Brasil sob o } \\
\text { aspecto de antecedentes históricos } \\
\text { e realizar revisão sistemática de } \\
\text { estudos publicados sobre registro } \\
\text { da vigilância do desenvolvimento } \\
\text { infantil e seus desafios encontrados } \\
\text { mediante aplicação da Caderneta } \\
\text { de Saúde da Criança. }\end{array}$ & $\begin{array}{l}\text { Fez-se busca da literatura em abril de } 2016 \text { nas bases } \\
\text { eletrônicas: Literatura Latino- Americana e do Caribe } \\
\text { em Ciências da Saúde (LILACS), Scientific Electronic } \\
\text { Library Online (SciELO) e Medical Literature } \\
\text { Analysis and Retrieval System Online (Medline), sem } \\
\text { restrição do idioma nem período de publicação, e em } \\
\text { referências bibliográficas dos artigos selecionados. }\end{array}$ & $\begin{array}{l}\text { Evidencia-se a atitude de descaso em relação à vigilância do } \\
\text { desenvolvimento infantil na atenção básica de saúde. Desafio } \\
\text { dos enfermeiros é fazer o preenchimento adequado e repassar } \\
\text { para os demais profissionais o uso correto da CSC para não a } \\
\text { ver nenhum esquecimento e erro que deveria ser prescrito na } \\
\text { mesma. É uma questão que deve ser levada aos fóruns } \\
\text { nacionais e internacionais como um problema de direitos } \\
\text { humanos, mesmo que a opinião pública ainda não se interessa } \\
\text { pelo assunto. }\end{array}$ \\
\hline $\begin{array}{c}3 \\
\text { BIREME }\end{array}$ & $\begin{array}{l}\text { Lima et } \\
\text { al. } \\
(2016)\end{array}$ & $\begin{array}{l}\text { Analisar artigos da literatura } \\
\text { nacional relativo à relevância da } \\
\text { utilização da Caderneta de Saúde da } \\
\text { Criança e seu preenchimento de } \\
\text { qualidade. }\end{array}$ & $\begin{array}{l}\text { Trata-se de uma revisão integrativa, realizada na } \\
\text { biblioteca virtual BIREME e na base de dados } \\
\text { LILACS, SCIELO e BDENF, em publicações com } \\
\text { data entre } 2005 \text { a } 2012 \text {, sendo analisados sete artigos } \\
\text { que compuseram a amostra dentro dos critérios } \\
\text { acatados no estudo. }\end{array}$ & $\begin{array}{l}\text { O resultado apresentado através desse estudo foi que A CSC é } \\
\text { um instrumento fundamental e indispensável para o } \\
\text { acompanhamento infantil. Percebe-se que, em geral, o } \\
\text { preenchimento da CSC necessita ser aprimorado, no intuito de } \\
\text { atingir a competência que lhe é devida. }\end{array}$ \\
\hline $\begin{array}{c}4 \\
\text { SCIELO }\end{array}$ & $\begin{array}{l}\text { Amorim } \\
\text { et al. } \\
(2016)\end{array}$ & $\begin{array}{l}\text { Avaliar o preenchimento da } \\
\text { Caderneta de Saúde da Criança } \\
\text { (CSC) e a associação entre } \\
\text { qualidade } \\
\text { preenchimento e o tipo de serviço } \\
\text { usado para o acompanhamento da } \\
\text { saúde das crianças. }\end{array}$ & $\begin{array}{l}\text { Estudo transversal com amostra estratificada e } \\
\text { proporcional aos 9 Distritos Sanitários de Belo } \\
\text { Horizonte, selecionada entre crianças de } 3 \text { a } 5 \text { anos, no } \\
\text { Dia da Campanha de Vacinação Infantil 2014. } \\
\text { Realizou-se entrevista com os pais e observação de } 21 \\
\text { itens da CSC. A variável dependente foi definida pela } \\
\text { qualidade (satisfatória /insatisfatória) do } \\
\text { preenchimento da CSC, sendo considerado satisfatório } \\
\text { o preenchimento }>60 \% \text {. }\end{array}$ & $\begin{array}{l}\text { Os resultados sugerem que há problemas na qualidade do } \\
\text { preenchimento da Caderneta de Saúde da Criança, } \\
\text { independentemente do tipo de serviço usado pelos pais para } \\
\text { acompanhamento dos seus filhos. A capacitação dos } \\
\text { profissionais envolvidos pode promover maior valorização do } \\
\text { instrumento, contribuindo para que a CSC cumpra seus } \\
\text { objetivos. }\end{array}$ \\
\hline
\end{tabular}




\begin{tabular}{|c|c|c|c|c|}
\hline $\begin{array}{c}5 \\
\text { SCIELO }\end{array}$ & $\begin{array}{l}\text { Vieira et } \\
\text { al. } \\
(2016)\end{array}$ & $\begin{array}{l}\text { Averiguar os fatores associados à } \\
\text { leitura da Caderneta de Saúde da } \\
\text { Criança (CSC) pelas mães e a seu } \\
\text { preenchimento pelos } \\
\text { profissionais de saúde, em Feira de } \\
\text { Santana (Bahia), em } 2009 \text {. }\end{array}$ & $\begin{array}{l}\text { Estudo transversal com aplicação de } 727 \text { formulários } \\
\text { às mães de crianças menores de um ano. Os desfechos } \\
\text { estudados foram: leitura materna da caderneta e } \\
\text { preenchimento de medidas de peso e comprimento, por } \\
\text { profissionais de saúde. Realizou-se análise de } \\
\text { regressão logística com valor de } \mathrm{p} \leq 5 \% \text {. }\end{array}$ & $\begin{array}{l}\text { Á uma grande necessidade de uma rede de articulação } \\
\text { interfederativa e intersetorial com os gestores municipais, para } \\
\text { ações de implementação de estratégias de orientações das mães } \\
\text { sobre a importância e o manejo da CSC, bem como de } \\
\text { qualificação e motivação dos profissionais de saúde, que } \\
\text { permitam uma reflexão sobre a prática desenvolvida frente a } \\
\text { um documento de reconhecida importância no } \\
\text { acompanhamento do CD e capacidade de pro- moção da saúde } \\
\text { infantil. }\end{array}$ \\
\hline $\begin{array}{c}6 \\
\text { PUBMED }\end{array}$ & $\begin{array}{l}\text { Almeida } \\
\text { et al. } \\
(2015)\end{array}$ & $\begin{array}{l}\text { Avaliar o uso de uma ferramenta } \\
\text { de monitoramento de saúde em } \\
\text { crianças brasileiras, com ênfase } \\
\text { sobre as variáveis relacionadas ao } \\
\text { crescimento e desenvolvimento. }\end{array}$ & $\begin{array}{l}\text { Foi realizada uma revisão sistemática da literatura em } \\
\text { estudos realizados no Brasil, utilizando os bancos de } \\
\text { dados Cochrane Brasil, Lilacs, SciELO e Medline. Os } \\
\text { estudos são selecionados por título e resumo e aqueles } \\
\text { considerados escolhidos na íntegra. }\end{array}$ & $\begin{array}{l}\text { Os resultados revelam a subutilização da fermentação e o } \\
\text { rebaixamento da conscientização dos dois profissionais de } \\
\text { saúde quanto ao registro de informações não no documento de } \\
\text { vigilância à saúde da criança. }\end{array}$ \\
\hline $\begin{array}{c}7 \\
\text { SCIELO }\end{array}$ & $\begin{array}{l}\text { Abud e } \\
\text { Gaiva } \\
(2015)\end{array}$ & $\begin{array}{l}\text { Analisar o preenchimento dos } \\
\text { dados de } \quad \text { crescimento } \\
\text { desenvolvimento na caderneta de } \\
\text { saúde da criança. }\end{array}$ & $\begin{array}{l}\text { Estudo transversal, realizado em Cuiabá, Brasil, em } 13 \\
\text { de agosto de 2011, com aplicação de formulário e } \\
\text { observação direta de } 950 \text { cadernetas. Foram incluídas } \\
\text { crianças menores de um ano, residentes em Cuiabá, } \\
\text { acompanhadas da mãe ou do responsável e de posse da } \\
\text { caderneta. Os dados foram analisados calculando-se a } \\
\text { razão de prevalência (RP) e teste Qui-Quadrado, com } \\
\text { nível de significância de 5\%. }\end{array}$ & $\begin{array}{l}\text { O baixo índice de preenchimento dos indicadores, crescimento } \\
\text { e desenvolvimento, reforça a necessidade de sensibilização da } \\
\text { população, de profissionais e gestores da saúde para a } \\
\text { importância da caderneta de saúde da criança, bem como } \\
\text { investimentos na formação e capacitação dos profissionais } \\
\text { quanto ao seu uso adequado. }\end{array}$ \\
\hline $\begin{array}{c}8 \\
\text { SCIELO }\end{array}$ & $\begin{array}{l}\text { Vieira et } \\
\text { al. } \\
(2017)\end{array}$ & $\begin{array}{l}\text { Analisar o registro de ações para a } \\
\text { prevenção da morbidade na } \\
\text { infância, a partir das informações } \\
\text { de vacinação, suplementação de } \\
\text { ferro e vitamina. }\end{array}$ & $\begin{array}{l}\text { Trata-se de um estudo transversal, com abordagem } \\
\text { quantitativa, realizado em Unidades de Saúde da } \\
\text { Família de João Pessoa-Paraíba, com amostragem por } \\
\text { conveniência, totalizando } 116 \text { cadernetas. Os dados } \\
\text { foram coletados a partir da observação dos registros } \\
\text { nas cadernetas e analisados conforme estatística } \\
\text { simples }\end{array}$ & $\begin{array}{l}\text { É notória a importância da CSC para a prevenção de agravos e } \\
\text { promoção da saúde, pois através dos registros é possível avaliar } \\
\text { as ações realizadas para a criança. Dessa forma, a caderneta } \\
\text { caracteriza-se como um instrumento aliado aos programas de } \\
\text { imunização e suplementação de ferro e vitamina A e, por isso, } \\
\text { deve ser incorporado à rotina das USF, também uma } \\
\text { ferramenta de avaliação da qualidade da atenção prestada. } \\
\text { Portanto, os profissionais de saúde que prestam assistência à } \\
\text { criança devem registrar as intervenções na caderneta em todos } \\
\text { os atendimentos, mesmo sendo um desafio, esse preenchimento } \\
\text { deve adequado deve acontecer por todos profissionais para que } \\
\text { possa acompanhar o estado de saúde da criança e, assim, } \\
\text { possam realizar um cuidado integral }\end{array}$ \\
\hline 9 & Costa et & proporção de & Estudo transversal de base populacional com crianças & Existe um baixo percentual de cadernetas de saúde \\
\hline
\end{tabular}




\begin{tabular}{|c|c|c|c|c|}
\hline SCIELO & $\begin{array}{c}\text { al. } \\
(2014)\end{array}$ & $\begin{array}{l}\text { crianças com cadernetas de saúde } \\
\text { com preenchimento adequado e } \\
\text { analisar associações com } \\
\text { características geográficas, } \\
\text { socioeconômicas e biológicas } \\
\text { maternas e da criança e com o } \\
\text { percentual de utilização de serviços } \\
\text { de saúde em dois municípios do } \\
\text { semiárido brasileiro. }\end{array}$ & $\begin{array}{l}\text { até cinco anos de idade. A coleta de dados foi realizada } \\
\text { mediante aplicação de questionários domiciliar entre } \\
\text { julho e setembro de 2008. A presença de todos os } \\
\text { registros na caderneta, relativos à identificação, } \\
\text { crescimento, desenvolvimento e status vacinal da } \\
\text { criança foi definida como preenchimento adequado. } \\
\text { Calcularam-se as razões de prevalência brutas e } \\
\text { ajustadas pela Regressão de Poisson, seguindo modelo } \\
\text { hierarquizado. }\end{array}$ & $\begin{array}{l}\text { adequadamente preenchidas. A maior prevalência de } \\
\text { cadernetas com preenchimento inadequado nas mães de baixa } \\
\text { escolaridade pode denotar iniquidade no sistema de saúde } \\
\text { local. O preenchimento adequado da caderneta um direito da } \\
\text { criança, a sua inadequação indicou despreparo dos } \\
\text { profissionais de saúde e dos pais às necessidades da atenção } \\
\text { básica. }\end{array}$ \\
\hline $\begin{array}{c}10 \\
\text { SCIELO }\end{array}$ & $\begin{array}{l}\text { Palombo } \\
\text { et al. } \\
(2014)\end{array}$ & $\begin{array}{l}\text { Avaliar uso e preenchimento da } \\
\text { Caderneta de Saúde da Criança } \\
\text { (CSC), especialmente crescimento } \\
\text { e desenvolvimento. }\end{array}$ & $\begin{array}{l}\text { Estudo transversal com } 358 \text { pares mãe- criança } \\
\text { atendidos em } 12 \text { Unidades Básicas de Saúde (UBS) de } \\
\text { município de pequeno porte. As mães foram } \\
\text { entrevistadas nas UBS, de fevereiro a abril de 2013, } \\
\text { com o uso de questionário. Utilizou-se softwares Epi- } \\
\text { info e Stata }\end{array}$ & $\begin{array}{l}\text { O uso e o preenchimento insatisfatório da CSC reforçam a } \\
\text { necessidade de investimentos na capacitação dos profissionais } \\
\text { e na sensibilização da comunidade para que a CSC se torne } \\
\text { efetivamente um instrumento de promoção da saúde infantil. }\end{array}$ \\
\hline
\end{tabular}

Fonte: Pesquisa direta (2020). 
A análise da caderneta de saúde da criança é de suma importância para rastreamento do desenvolvimento infantil na atenção primaria à saúde e a observar a qualidade do preenchimento; visitas realizadas nas primeiras semanas aos recémnascidos contribuem para identificação de possíveis alterações que possam implicar diretamente no desenvolvimento infantil e rastreamento do TEA, o que pode ser evidenciado com os desfechos apresentado no Quadro 3.

Diversos são os problemas inerentes ao acompanhamento do desenvolvimento infantil e suas repercussões manifestam-se durante toda a vida, principalmente, quando estes têm alguma alteração psicológica (Almeida et al., 2015; Lima, Nobre, Lopes, Rolim, Albuquerque, \& Araujo, 2016).

Muitos autores descrevem que os tabus e dificuldades no acompanhamento do desenvolvimento infantil relacionam-se com a prática de enfermagem e pode ser minorada por dificuldades do profissional em reconhecer as alterações e por deficiência de conhecimentos teóricos (Abud \& Gaiva, 2015; Caminha, Silva, Lima, Azevedo, Figueira, \& Batista Filho, 2017).

O autismo é considerado como uma condição derivada de um grupo de desordens complexas do desenvolvimento do cérebro, esses distúrbios se identificam pela dificuldade na comunicação social e comportamentos repetitivos e devem ser identificados durante o acompanhamento da carteira de saúde da criança (Vieira et al., 2016; Amorim et al., 2016; Vieira et al., 2017).

De modo geral todos os artigos abordavam as dificuldades e a subutilização da ferramenta que refletem na baixa conscientização dos profissionais de saúde sobre o registro de informações no documento de monitoramento de saúde da criança (Costa et al., 2014; Palombo, Duarte, Fujimori, Tamami, \& Toriyama, 2014; Amorim et al., 2018).

\section{Principais problemas relacionados ao preenchimento inadequado das cadernetas de saúde da criança}

O estudo Realizado por Amorim et al. (2018), teve como objetivo descrever o preenchimento da Caderneta de Saúde da Criança (CSC) nos serviços de saúde, onde o mesmo foi desenvolvido em Belo Horizonte e observou como como principais resultados que o enfrentamento da precariedade nos registros da CSC passa pela formação de profissionais de saúde, no qual eles devem reconhecer a importância do instrumento para a vigilância da saúde infantil, bem como do empoderamento dos pais ou responsáveis, que devem portar e exigir o uso da CSC nos diferentes serviços de saúde da criança.

Já o estudo Caminha et al. (2017), vem justificando que no Brasil, é preciso observar os aspectos de antecedentes históricos que acompanham a vigilância do desenvolvimento infantil e os desafios encontrados mediante aplicação da Caderneta de Saúde da Criança. Esse estudo corrobora com a pesquisa de Souza, Pereira, Silva e De Paula (2019), que o aborda os desafios dos enfermeiros em fazer o preenchimento adequado e repassar para aos demais profissionais o uso correto da CSC para que não haja erros futuros e nenhum esquecimento de algo que deveria ser prescrito na mesma.

Segundo Carvalho-Filha, Moraes-Filho, Santos, Silva e Pereira (2018), o conhecimento de enfermeiros sobre o acompanhamento do desenvolvimento infantil por meio da caderneta de saúde da criança é indispensável no atendimento ou atenção à saúde da população em geral, e o seu conhecimento teórico-prático e clínico.

Nascimento, Castro, Lima, Albuquerque, e Bezerra, (2018), destacam que, para que haja o desempenho correto das suas funções, o enfermeiro deve ter maior preparo clínico e científico, buscando a resolutividade de forma ampliada na assistência de enfermagem à família e à comunidade.

Neste aspecto, Vieira et al. (2016) afirmam que as mães muitas vezes, não reconhecem a necessidade de sua participação na avaliação do crescimento e desenvolvimento de seus filhos e não tem interesse pelo cartão da criança, pois, habitualmente, as ações de saúde sempre foram delegadas aos profissionais, não sendo permitida ou estimulada a interferência ativa das mães ou familiares nesse processo. 
Para Della Barba, Barros, Figueiredo e Martinez (2016), os profissionais têm uma grande dificuldade em manejar a caderneta de saúde da criança, encontrando-se despreparados para prestar uma vigilância à saúde da criança com eficácia. Sendo indispensável investir em mais capacitação para os profissionais com a finalidade de despertar a consciência destes para a importância do conhecimento da caderneta e reconhecimento de sinais que indiquem o Transtorno do Espectro Autista.

Corroborando com os demais autores, Amorim et al. (2016) e Vieira et al. (2016), afirmam que a atenção à saúde de crianças com deficiência ou em situações específicas e de vulnerabilidade: consiste na articulação de um conjunto de estratégias intrassetoriais e intersetoriais, para inclusão dessas crianças nas redes temáticas de atenção à saúde, mediante a identificação de situação de vulnerabilidade e risco de agravos e adoecimento, reconhecendo as especificidades deste público para uma atenção resolutiva.

Segundo o estudo de Silva et al. (2020) há inúmeros benefícios no uso de intervenção precoce em crianças com transtorno do espectro autista, entre eles destacam-se: Melhor desenvolvimento cognitivo e maior aprendizagem, redução do estresse para a criança e familiares e, maior interação social. Neste aspecto, ressalta-se a necessidade dos profissionais de enfermagem identificar ou colaborarem para identificação dessas crianças.

Onde a pesquisa de Caminha et al. (2017), e Lima et al. (2016), destacam algumas variáveis previamente associadas à má qualidade do preenchimento da CSC mostrando associação com o presente estudo, são eles: idade da mãe, escolaridade, renda, número de gestações. Evidenciando que mães mais jovens e em condições de desvantagem social (residência em comunidades mais pobres, mães de famílias grandes, baixa escolaridade da mãe) acabam não se atendando a algumas particularidades dos filhos, colaborando para o diagnostico tardio de alguns transtornos, como é o caso do TEA.

Por fim, os autores Amorim et al. (2018) e Almeida et al. (2015), e Abud e Gaiva (2015) apontam que os serviços de saúde muitas vezes não alcançam os objetivos propostos. Evidenciando deficiências nas ações de saúde, pois se a atenção fosse realmente continuada e integral, tanto as mães que realizaram pré-natal quanto mães que foram mais resistentes, seriam sensibilizadas quanto a importância do atendimento a seus filhos, especialmente durante o primeiro ano de vida.

\section{Considerações Finais}

A Caderneta de Saúde da Criança é um instrumento fundamental para que os profissionais da saúde possam acompanhar o crescimento e o desenvolvimento físico e mental das crianças, podendo ser utilizado no rastreamento de doenças e inclusive, no rastreamento de TEA. Onde a indispensabilidade da utilização desta ferramenta é nítida para a vigilância da saúde da criança, uma vez que, sua utilização de modo adequado e disciplinado, oferece informações precisas sobre o estado físico e mental das crianças.

Onde o estudo evidenciou os problemas que influenciam o preenchimento inadequado das cadernetas pelos profissionais de saúde, com destaque para: dificuldade dos profissionais de perceberem a relevância do preenchimento, ao conhecimento insatisfatório por parte desses profissionais, além da carência de orientações às famílias. Quanto às limitações deste estudo citam-se o fato de que poucos estudos e bases de dados abordam a temática sobre desafios na utilização da caderneta de saúde da criança para o diagnóstico de crianças com transtorno do espectro autista.

Por fim, a pesquisa colaborou para o aperfeiçoamento do conhecimento e compreensão sobre a saúde da criança e saúde da família além de a profundar os conhecimentos sobre o Transtorno do Espectro Autista e a forma correta de preencher a CSC. Portanto, há uma necessidade de instruir profissionais e a coletividade sobre os benefícios da utilização da CSC além da realização de mais pesquisas nesta linha de investigação para comprovar a importância de uma atenção especial a saúde da criança. 
Research, Society and Development, v. 10, n. 10, e126101018663, 2021 (CC BY 4.0) | ISSN 2525-3409 | DOI: http://dx.doi.org/10.33448/rsd-v10i10.18663

\section{Referências}

Abud, S. M., \& Gaíva, M. A. M. (2016). Analise do preenchimento dos dados sobre gravidez, parto, puerpério e recém-nascido na caderneta de saúde da criança. Rev Soc Bras Enferm Ped, 16(1), 11-20.

Almeida, J. H. H. D., Feitosa, A. N. A., Araújo, W. A., Silva, J. B., Lourenço, L. C., \& Sousa, M. N. A. (2015). Atenção primária à saúde: enfocando as redes de atenção à saúde. Revista de Enfermagem UFPE on line, 9(11), 9811-16.

Amorim, L. D. P., Senna, M. I. B., Gomes, V. E., Amaral, Vasconcelos, M., Silva, A. G. D., \& Ferreira, R. C. (2018). Preenchimento da Caderneta de Saúde da Criança nos serviços de saúde em Belo Horizonte, Minas Gerais, Brasil. Epidemiol. Serv. Saude, Brasília, 27(1), e201701116.

Amorim, L. D. P., Senna, M. I. B., Soares, A., R. C. S., Carneiro, G. T. N., Ferreira, E. F. E., Vasconcelos, M., ... \& Ferreira, R. C. (2016). Avaliação do preenchimento da Caderneta de Saúde da Criança e qualidade do preenchimento segundo o tipo de serviço de saúde usado pela criança. Ciência \& Saúde Coletiva, 23(2), 585-597.

Caminha, M. D. F. C., Silva, S. L. D., Lima, M. D. C., Azevedo, P. T. A. C. C. D., Figueira, M. C. D. S., \& Batista Filho, M. (2017). Vigilância do desenvolvimento infantil: análise da situação brasileira. Rev Paul Pediatr, 35(1), 102-109.

Carvalho-Filha, F. S. S., Moraes-Filho, I. M. D., Santos, J. C. D., Silva, M. V. D. R. S. D., \& Pereira, N. D. (2018). Entendimento do espectro autista por pais/cuidadores-estudo descritivo. Revista de Divulgação Científica Sena Aires, 7(2), 105-116.

Costa, J. S. D. D., Cesar, J. A., Patussi, M. P., Fontoura, L. P. D., Bazarreti, L., Nunes, M. F., \& Uebel, R. (2014). Assistência à criança: preenchimento da caderneta de saúde em municípios do semi-árido brasileiro. Rev. Bras. Saúde Matern. Infant., 14 (3), 219-227.

Della Barba, P.C.S., Barros, V. D. M., Figueiredo, M. O., \& Martinez, L. B. A. (2016). Formação em vigilância do desenvolvimento infantil na atenção primária à saúde. Revista Brasileira de Iniciação Científica, 4(2), 1-19.

Fernandes, M. A., Vieira, F. M. R., Silva, J. F., Avelino, F. V. S. D., \& Santos, J. D. M. (2018). Prevalência de sintomas ansiosos e depressivos em universitários de uma instituição pública. Revista Brasileira de Enfermagem, 71, 2169-2175.

Lima, L. G., Nobre, C. S., Lopes, A. C. M. U., Rolim, K. M. C. Albuquerque, C. D. M., \& Araujo, M. A. L. (2016). A Utilização da caderneta de saúde da criança no acompanhamento infantil. Rev. bras. ciênc. saúde, 20(2), 167-174.

Melo, M. M., Fontes, F. L. D. L., Sousa, E. L. D, Sousa, E. A. E, Nascimento, F. S. D, Andrade, G. B. M. D., \& OliveiraE. S. B. de. (2019). Atendimento multidisciplinar para a educação especial e inclusiva de uma criança com transtorno do espectro autista: um estudo de caso. Revista Eletrônica Acervo Saúde, 25 , e589.

Nascimento, Y. C. M. L., Castro, C. S. C. D., Lima, J. L. R. D., Albuquerque, M. C. D. S. D., \& Bezerra, D. G. (2018). Transtorno do espectro autista: detecção precoce pelo enfermeiro na Estratégia Saúde da Família. Rev. baiana enferm, 32, e25425.

Palombo, C. N. T., Duarte, L. C., Fujimori, E., Tamami, A., \& Toriyama, M. (2014). Uso e preenchimento da caderneta de saúde da criança com foco no crescimento e desenvolvimento. Revista Esc. Enferm. USP, 48, 59-66.

Paiva, M. R. F., Parente, J. R. F., Brandão, I. R., \& Queiroz, A. H. B. (2016). Metodologias ativas de ensino-aprendizagem: revisão integrativa. SANARERevista de Políticas Públicas, 15(2), 145-153

Rosolem, L. H., Toninato, A. P. C., Sanguino, G. Z., Bonati, P. C. D. R., Rezende, V. D., Mello, D. F. D., \& Furtado, M. C. D. C. (2019). Caderneta de saúde da criança: coordenação do cuidado e acesso à saúde. Cogitare Enfermagem, 24, e61496.

Silva, C. O. D., Oliveira, S. A., Silva, W. C. D., Mendes, R. C., Miranda, L. S. C., Melo, K. C., \& Santos, M. E D. J. (2020). Benefícios no uso de intervenção precoce em crianças com transtorno do espectro autista (TEA): uma revisão integrativa. Research, Society and Development, 9(7), e256972474.

Silva, J., Hoyos, S. E. V., Oliveira, A. A. D., Moura, S. O. D., \& Kortmann, G. M. L. (2018). Diagnóstico do autismo na primeira infância. Anais da Semana Científica da Unilasalle (SEFIC), Canoas, SC, Brasil.

Souza, N. S., Pereira, L. P. S., Silva, S. V., De Paula, \& W. K. A. S. (2019). Vigilância e estímulo do crescimento e desenvolvimento infantil. Rev enferm UFPE on line, 13(3), 680-9.

Urbanetto, J. S., Freitas, A. P. C. D., Oliveira, A. P. R. D., Santos, J. D. C. R. D., Muniz, F. D. O. M., Silva, R. M. D., \& Schilling, M. C. L. (2017). Fatores de risco para o desenvolvimento de flebite: uma revisão integrativa da literatura. Revista Gaúcha de Enfermagem, 38(4), e57489.

Vieira, D. D. S., Santos, N. C. C. D. B., Costa D. K. G. B., Pereira, M. D. M., Vaz, E. M. C., Reichert, A. P. D. S. (2016). Registro de ações para prevenção de morbidade infantil na caderneta de saúde da criança. Ciência \& saúde. 21(7), 2305- 2313.

Vieira, G. O., Bastos, M. C., Reis, M. R. D., Moreira, I. S. S., Martins, C. D. C., Gomes, D. R., \& Vieira, T. D. O. (2017). Fatores associados ao uso da Caderneta de Saúde da Criança em uma cidade de grande porte do nordeste brasileiro, 2009. Ciência \& saúde. 23(6), 1943-1954. 